\title{
An association study between various monoamine transporter gene polymorphisms and treatment response to mirtazapine in major depression
}

Hong Choi

\author{
From $1^{\text {st }}$ International Congress on Neurobiology and Clinical Psychopharmacology \\ and European Psychiatric Association Conference on Treatment Guidance \\ Thessaloniki, Greece. 19-22 November 2009
}

\section{Background}

Genetic differences may contribute to the inter-individual differences in treatment response to antidepressants among patients suffering from major depression. This study investigated a possible association of various monoamine transporter genetic polymorphisms with treatment response to mirtazapine in major depressive patients in elderly.

\section{Materials and methods}

In this study, three genetic polymorphisms were selected: serotonin transporter 5-HTTLPR, serotonin transporter 5-HTT intron 2 VNTR, and norepinephrine transporter $\operatorname{NET}($ G1287A). The patients with major depression diagnosed by DSM-IV were recruited to a 6-week naturalistic mirtazapine treatment study in Samsung Medical Center. Treatment response to mirtazapine was defined as $\geq 50 \%$ decrease in HAMD- 17 scores at 6 weeks, and the genotypes in the patients were determined using the polymerase chain reaction.

\section{Results}

Our results showed that ss allele carriers were included more in responder group(ss allele in responder vs. non responder group; $69.4 \%$ vs. $40.0 \%$ ). In addition, l-allele $(\mathrm{sl} / \mathrm{ll})$ carriers were included less in responder group(sl/ll allele in responder vs. non responder group; $30.6 \%$ vs. $60.0 \%$ ). Multiple logistic regression analyses showed the 5-HTTLPR polymorphism as an predictor of the mirtazapine response (5HTTLPR ss allele carrier vs. 1-allele (sl/ll) carrier; odds ratio: 3.81; 95\% confidence interval $[\mathrm{CI}], 1.32-11.0 ; \mathrm{P}=0.013)$. However, 5-HTT intron 2 VNTR $1 / \mathrm{s}(\mathrm{P}=0.33$ by multiple logistic regression; [OR], 0.53; 95\% [CI], 0.15-1.88), and NET (G1287A) G/A ( $\mathrm{P}=0.68$ by multiple logistic regression; [OR], 1.25; 95\% [CI], 0.44-3.53) showed no statistical significant influences on response rate.

\section{Conclusions}

In conclusion, 5HTTLPR polymorphism may predict treatment response to mirtazapine in major depressive patients in elderly.

Published: 22 April 2010

doi:10.1186/1744-859X-9-S1-S203

Cite this article as: Choi: An association study between various monoamine transporter gene polymorphisms and treatment response to mirtazapine in major depression. Annals of General Psychiatry 2010 9(Suppl 1):S203. 